\title{
Model risk in mean-variance portfolio selection: an analytic solution to the worst-case approach
}

\author{
Roberto Baviera ${ }^{1}$ (D) $\cdot$ Giulia Bianchi ${ }^{1}$
}

Received: 9 December 2019 / Accepted: 16 May 2021 / Published online: 29 May 2021

(c) The Author(s) 2021

\begin{abstract}
In this paper we consider the worst-case model risk approach described in Glasserman and Xu (Quant Finance 14(1):29-58, 2014). Portfolio selection with model risk can be a challenging operational research problem. In particular, it presents an additional optimisation compared to the classical one. We find the analytical solution for the optimal mean-variance portfolio selection in the worst-case scenario approach and for the special case with the additional constraint of a constant mean vector considered in Glasserman and Xu (Quant Finance 14(1):29-58, 2014). Moreover, we prove in two relevant cases-the minimum-variance case and the symmetric case, i.e. when all assets have the same mean - that the analytical solutions in the alternative model and in the nominal one are equal; we show that this corresponds to the situation when model risk reduces to estimation risk.
\end{abstract}

Keywords Model risk · Robust portfolio selection · Mean-variance portfolio · Kullback-Leibler divergence

JEL Classifications C51 · D81 - G11

\section{Notation}

1

A, B, C, D

$\alpha$

$\mathbf{a}$

$\mathbf{a}_{\text {nom }}^{\star}, \mathbf{a}^{\star}(\theta)$

$\mathcal{A}$

$\gamma$

$\Gamma(S ; \theta, \gamma)$

$\Gamma^{G X}(S ; \theta, \gamma)$

$d, d^{\star}$

$\mathcal{D}$
Vector of all $1 \mathrm{~s}$ in $\mathbb{R}^{n}$

Defined in (7)

Lagrange multiplier

Portfolio's weights vector, named portfolio

Optimal portfolio in the nominal measure and in the alternative measure

Domain for the portfolio a, satisfying the constraint $\mathbf{1}^{T} \mathbf{a}=1$

Risk aversion parameter

Defined in (20)

Defined in (36)

Dual Lagrangian problem corresponding to $p$ and related optimal value Domain for $(\theta, \mathbf{a})$ defined in (15)

Roberto Baviera

roberto.baviera@polimi.it

1 Department of Mathematics, Politecnico di Milano, 32 p.zza Leonardo da Vinci, 20133 Milan, Italy 


\begin{tabular}{|c|c|}
\hline$f(\mathbf{X}), \tilde{f}(\mathbf{X})$ & Probability densities in nominal and alternative model \\
\hline$\eta$ & Maximum KL-divergence between the alternative and the nominal one \\
\hline$\theta, \theta^{\star}$ & Optimisation parameter and corresponding optimum \\
\hline$\theta_{\max }(\mathbf{a})$ & Upper bound for $\theta$ defined in (12) \\
\hline$\tilde{\theta}$ & $\operatorname{Argmin}$ in $\theta$ of $\mathcal{L}(\theta, \mathbf{a})$ for a given portfolio $\mathbf{a}$ \\
\hline$I$ & Identity matrix \\
\hline$k$ & Multiplicative parameter of the variance parameter $\sigma^{2}$ \\
\hline$\lambda_{i}, \tilde{\lambda}_{i}$ & Eigenvalues of the variance matrices $\Sigma$ and $\tilde{\Sigma}$ \\
\hline $\mathcal{L}(\theta, \mathbf{a}, m(\mathbf{X}))$ & $\begin{array}{l}\text { Lagrangian function associated to constrained maximisation problem } \\
\text { (3) }\end{array}$ \\
\hline $\mathcal{L}(\theta, \mathbf{a})$ & Lagrangian function computed in the optimal change of measure (10) \\
\hline$m(\mathbf{X})$ & Change of measure, defined as $\tilde{f}(\mathbf{X}) / f(\mathbf{X})$ \\
\hline$m_{\theta, \mathbf{a}}^{\star}(\mathbf{X})$ & Worst-case change of measure depending on parameter a \\
\hline$m_{\theta}^{\star}(\mathbf{X})$ & $\begin{array}{l}\text { Worst-case change of measure corresponding to the optimal portfolio } \\
\mathbf{a}^{\star}(\theta)\end{array}$ \\
\hline$m^{\star p}(\mathbf{X}), m_{\theta^{\star}}^{\star d}(\mathbf{X})$ & Optimal change of measures for the primal and dual problem $p$ and $d$ \\
\hline$\mu, \tilde{\mu}$ & Mean vector in the nominal and in the alternative model \\
\hline$n$ & Number of assets considered \\
\hline$p, p^{\star}$ & Primal problem optimisation in (3) and related optimal value \\
\hline$P_{\eta}$ & KL-ball with radius $\eta$ \\
\hline$\rho$ & Correlation parameter \\
\hline$R(\tilde{f}, f)$ & Relative entropy function between nominal and alternative models \\
\hline$R(\theta, \mathbf{a})$ & Relative entropy corresponding to the optimal change of measure (10) \\
\hline$R(\theta)$ & Relative entropy corresponding to the optimal portfolio $\mathbf{a}^{\star}(\theta)$ \\
\hline$S, S^{\star}(\theta)$ & Defined as $\mathbf{a}^{T} \sum \mathbf{a}$ for a portfolio $\mathbf{a}$ and for the optimal one $\mathbf{a}^{\star}(\theta)$ \\
\hline$\Sigma, \tilde{\Sigma}$ & Variance matrix in the nominal and in the alternative model \\
\hline$\sigma^{2}$ & Variance parameter \\
\hline$V_{\mathbf{a}}(\mathbf{X})$ & Measure of risk associated with $\mathbf{X}$ and with parameter $\mathbf{a}$ \\
\hline$V_{\mathbf{a}}^{G X}(\mathbf{X})$ & Measure of risk in the special case with constant mean \\
\hline $\mathbf{X}$ & Stochastic asset returns \\
\hline
\end{tabular}

\section{Introduction}

Markowitz [12] was the first to introduce an optimal portfolio selection according to the mean and the variance. Since that seminal paper, this problem has been extensively studied (see e.g. [11], and references therein). This criterion is at the base of modern portfolio theory and it is widely used in finance due to its simplicity given that it models asset returns as Gaussian random variables.

The accuracy of this portfolio selection crucially depends on the reliability of this model, which is named nominal model. Model risk is the risk arising from using an insufficiently accurate model. A quantitative approach to model risk is the worst-case approach, which was introduced in decision theory by Gilboa and Schmeidler [4]. According to this methodology, one considers a class of alternative models and minimises the loss encountered in the worstcase scenario.

The literature distinguishes between estimation and misspecification risk (see e.g. [8])]. In general, it is interesting to identify vulnerabilities to model error that result not only from parameter perturbations (estimation risk) but also from an error in the joint distribution of 
returns (misspecification risk). The deviation between statistical distributions can be measured by the Kullback and Leibler [9] relative entropy, which is also known as KL divergence, as proposed by Hansen and Sargent [6] in the context of model risk. The problem of determining an optimal robust portfolio under KL divergence has been studied by Calafiore [2]; he proposed two numerical schemes to find an optimal portfolio in the mean-variance and the mean-absolute deviation cases, considering a discrete setting. This approach has been studied by Glasserman and $\mathrm{Xu}$ [5] in a continuous setting in a mean-variance case; the authors identified the worst-case alternative models to the nominal model and numerically found the optimal portfolio selection in these cases. More recently, Penev et al. [14] have analyzed the mean-standard deviation case in detail showing that this case presents a semi-analytic solution.

Let us briefly summarise the portfolio selection problem in presence of model risk. Let $\mathbf{X} \in \mathbb{R}^{n}$ denote the stochastic asset returns. The p.d.f. associated with $\mathbf{X}, f(\mathbf{X})$, corresponds to the nominal model, while the p.d.f. $\tilde{f}(\mathbf{X})$ corresponds to the alternative model. The KL divergence between the two models is

$$
R(\tilde{f}, f):=\mathbb{E}[m(\mathbf{X}) \ln m(\mathbf{X})]
$$

where $m(\mathbf{X}):=\tilde{f}(\mathbf{X}) / f(\mathbf{X})$ is the change of measure and $\mathbb{E}[\bullet]$ denotes the expectation w.r.t. $f(\mathbf{X})$. In particular, we are interested in the alternative models within a ball $P_{\eta}$ of radius $\eta>0$ around the nominal model (KL-ball), i.e., characterised by a KL divergence lower or equal to $\eta$.

Let $V_{\mathbf{a}}(\mathbf{X})$ denote a measure of risk associated with $\mathbf{X}$, that depends on the portfolio weights a ranging over a set $\mathcal{A}$; the classical optimal portfolio selection problem is

$$
\inf _{\mathbf{a} \in \mathcal{A}} \mathbb{E}\left[V_{\mathbf{a}}(\mathbf{X})\right]
$$

while the worst-case portfolio selection corresponds to

$$
\inf _{\mathbf{a}} \sup _{m \in P_{\eta}} \mathbb{E}\left[m(\mathbf{X}) V_{\mathbf{a}}(\mathbf{X})\right]
$$

It can be shown that it is equivalent to the dual problem (see e.g. [1])

$$
\inf _{\mathbf{a}} \inf _{\theta>0} \sup _{m} \mathcal{L}(\theta, \mathbf{a} ; m(\mathbf{X}))
$$

where

$$
\mathcal{L}(\theta, \mathbf{a} ; m(\mathbf{X}))=\mathbb{E}\left[m(\mathbf{X}) V_{\mathbf{a}}(\mathbf{X})-\frac{1}{\theta}(m(\mathbf{X}) \ln m(\mathbf{X})-\eta)\right]
$$

is the Lagrangian function associated to the constrained maximisation problem in (3).

Thus, in the worst-case portfolio selection, one has to solve three nested optimisation problems where the inner problem is an infinite dimensional optimisation. While the inner optimisation problem is a standard one in functional analysis and a closed form solution can be found (see e.g. [10]), the presence of the other two makes the optimal selection a challenging operational research problem. Glasserman and $\mathrm{Xu}$ [5] propose a numerical approach to solve 
this problem. ${ }^{1}$ In this study, we provide an analytical solution and we show that the problem can be challenging from a numerical point of view.

This paper makes three main contributions. First, we analytically solve the model risk optimisation problem in the worst-case approach when asset returns are Gaussian. This result is achieved for a class of problems that are even wider than those solved numerically by Glasserman and $\mathrm{Xu}$ [5]. In particular, we consider

- A generic mean-variance selection, and not just the case where we impose the additional constraint of the worst-case mean equal to the nominal one (cf. [5, p. 36]);

- All possible values of $\theta$, which allow a well-posed problem and we do not limit the analysis to " $\theta>0$ sufficiently small" (cf. [5, p. 31]); i.e., we do not consider only small balls $P_{\eta}$.

Second, we provide the solution also in the special case where we impose the additional constraint of constant mean in the alternative model: this is the optimization problem considered by [5, cf. Eq.(30), p. 36].

Third, we prove that, in the minimum-variance case and in the symmetric case with equal mean values for all assets in the portfolio, the optimal worst-case portfolio is the same as the optimal nominal portfolio. Moreover, we prove that in these cases model risk and estimation risk coincide: we show that any alternative model within the ball $P_{\eta}$ can be obtained through a parameter change. This result is different from the numerical solution in [5, Figure 1, p. 37].

The rest of this paper is structured as follows. In Sect. 2, we recall the problem formulation. In Sect. 3, we present model risk analytical solution in the mean-variance framework. In Sect. 4, we study in detail the case of mean-variance with fixed mean considered by Glasserman and $\mathrm{Xu}$ [5]. In Sect. 5, we focus on the case where the optimal portfolio in the alternative model and the one in the nominal model coincide and provide numerical examples. Section 6 concludes this paper.

\section{Problem formulation}

In this section we recall the worst-case approach for model risk. Let $\mathbf{X}$ denote the stochastic element of a model and a the parameters' vector ranging over the set $\mathcal{A}$; the nominal model corresponds to solve the optimisation problem (2) in the nominal measure, while the alternative model corresponds to the same problem with respect to an alternative measure, chosen within a KL-ball $P_{\eta}$ with $R(\tilde{f}, f)<\eta$; i.e., within all models with a KL-divergence from the nominal model lower than a positive constant $\eta$. In the best-case and in the worst-case approaches, the optimisation problem becomes

$$
\left\{\begin{array}{l}
\underset{\mathbf{i n f}}{\operatorname{anf}} \inf \mathbb{} \mathbb{E}\left[m(\mathbf{X}) V_{\mathbf{a}}(\mathbf{X})\right] \text { best-case } \\
\text { inf } \sup _{\mathbf{a}} \mathbb{E}\left[m(\mathbf{X}) V_{\mathbf{a}}(\mathbf{X})\right] \text { worst-case. }
\end{array}\right.
$$

In portfolio selection, to have a robust measure, we are more interested in the worst-case approach, so hereafter we focus, unless stated differently, on this case that corresponds to

1 It can be useful to mention that the scope of the work of Glasserman and Xu is far beyond the robust portfolio selection described here: they were the first who introduced worst-case model risk in a general continuous time setting through relative entropy. They have also considered a set of illustrative applications of the proposed model risk methodology: besides the robust mean-variance portfolio selection we consider in this paper, they have described other four possible applications (expected shortfall computation, multi-name credit risk, delta hedging error and credit valuation adjustment). 
the highest possible value of the measure of risk; mutatis mutandis similar results hold in the other case.

The rest of this section is organised as follows. First, to clarify the notation used in the case of interest, we summarise the classical mean-variance portfolio theory with its main results $[12,13]$. Then, we sum up the main results for the worst-case model risk approach in a rather general setting, following Glasserman and $\mathrm{Xu}$ [5] notation.

\subsection{Classical portfolio theory}

In this study, the nominal model is characterised by $n$ risky securities that are modeled as a vector of asset returns $\mathbf{X} \in \mathbb{R}^{n}$ distributed as a multivariate normal $\mathbf{X} \sim N(\boldsymbol{\mu}, \Sigma)$, with $\Sigma \in \mathbb{R}^{n \times n}$ a positive definite matrix with strictly positive diagonal elements. Let $\mathbf{a}$ be the vector of portfolio weights, defined in the set $\mathcal{A}:=\left\{\mathbf{a}: \mathbf{a}^{T} \mathbf{1}=1\right\}$, where $\mathbf{1}$ is the vector in $\mathbb{R}^{n}$ of all $1 \mathrm{~s}$.

In the mean-variance framework, one considers a quadratic measure of risk; i.e., the difference between the variance (multiplied by $\gamma$, a positive risk aversion parameter) and the expected return of the portfolio

$$
V_{\mathbf{a}}(\mathbf{X}):=\frac{\gamma}{2} \mathbf{a}^{T}(\mathbf{X}-\boldsymbol{\mu})(\mathbf{X}-\boldsymbol{\mu})^{T} \mathbf{a}-\mathbf{a}^{T} \mathbf{X}, \quad \gamma>0 .
$$

The value of the risk measure is

$$
\mathbb{E}\left[V_{\mathbf{a}}(\mathbf{X})\right]=\frac{\gamma}{2} \mathbf{a}^{T} \Sigma \mathbf{a}-\mathbf{a}^{T} \boldsymbol{\mu} .
$$

The problem consists of minimising the value of the risk measure on all portfolios a with weights summing to 1 . Using a Lagrange multiplier, the mean-variance portfolio selection problem can be written as

$$
\min _{\mathbf{a}}\left\{\frac{\gamma}{2} \mathbf{a}^{T} \Sigma \mathbf{a}-\mathbf{a}^{T} \boldsymbol{\mu}+\alpha\left(1-\mathbf{a}^{T} \mathbf{1}\right)\right\}
$$

where $\alpha$ is the multiplier.

Following Merton [13], we introduce the notation

$$
A:=\mathbf{1}^{T} \Sigma^{-1} \boldsymbol{\mu} \quad B:=\boldsymbol{\mu}^{T} \Sigma^{-1} \boldsymbol{\mu} \quad C:=\mathbf{1}^{T} \Sigma^{-1} \mathbf{1} \quad D:=B C-A^{2} ;
$$

it is straightforward to show that $B, C>0$ and $D \geq 0$ (see e.g. [13]).

The optimal mean-variance portfolio (see e.g. [13, Equation (9), p. 1854]) is

$$
\mathbf{a}_{\text {nom }}^{\star}=\frac{A}{\gamma} \frac{\Sigma^{-1} \boldsymbol{\mu}}{A}+\left(1-\frac{A}{\gamma}\right) \frac{\Sigma^{-1} \mathbf{1}}{C} .
$$

Any optimal portfolio $\mathbf{a}_{\text {nom }}^{\star}$ is the linear combination of two portfolios in the optimal frontier $\mathbf{a}_{1}^{\star}:=\Sigma^{-1} \boldsymbol{\mu} / A$ and $\mathbf{a}_{0}^{\star}:=\Sigma^{-1} \mathbf{1} / C$, where the latter is the portfolio of minimum variance. This important result is also known as the two mutual fund theorem.

\subsection{Worst case model risk}

We briefly recall the model risk formulation for the construction of the alternative model. In particular, we focus on the worst-case portfolio selection (3); i.e., the one that considers the maximum value of the risk measure within the KL-ball $P_{\eta}$. This worst-case problem is equivalent to the dual problem (4) with $V_{\mathbf{a}}(\mathbf{X})$ defined in (5); mutatis mutandis an analogous result holds in the best-case with $\theta<0$. 
Remark 1 Glasserman and Xu [5] consider the special case with the additional constraint $\boldsymbol{\mu}=\mathbb{E}[m(\mathbf{X}) \mathbf{X}]$; in their case, it is equivalent to consider, instead of (5), the measure of risk

$$
V_{\mathbf{a}}^{G X}(\mathbf{X}):=\frac{\gamma}{2} \mathbf{a}^{T}(\mathbf{X}-\boldsymbol{\mu})(\mathbf{X}-\boldsymbol{\mu})^{T} \mathbf{a}-\mathbf{a}^{T} \boldsymbol{\mu}, \quad \gamma>0 .
$$

In Sect 4, we show that all results obtained in the mean-variance framework hold even in this special case.

Thus, we have to consider the three nested optimisation problems in (4). The inner optimisation problem is standard in functional analysis. For a given $\theta>0$ and for a given $\mathbf{a} \in \mathcal{A}$, the solution of the internal maximisation problem on the variable $m(\mathbf{X})$ in (4) is

$$
m_{\theta, \mathbf{a}}^{\star}(\mathbf{X})=\frac{\exp \left(\theta V_{\mathbf{a}}(\mathbf{X})\right)}{\mathbb{E}\left[\exp \left(\theta V_{\mathbf{a}}(\mathbf{X})\right)\right]} .
$$

This result is known in the literature (see e.g. [5,6]). For a complete proof, the interested reader can refer to [10, Proposition 3.1]. Unfortunately, the other two optimisations are more challenging and closed form solutions cannot be found in the literature for the case of interest.

Before entering into the details of the two optimisations in a and $\theta$, it is interesting to observe some properties for the entropy computed on the optimal solution of the internal maximisation problem

$$
R(\theta, \mathbf{a}):=R\left(f m_{\theta, \mathbf{a}}^{\star}, f\right)
$$

where $R(\tilde{f}, f)$ is defined in (1). They are stated in the following lemma.

Lemma 1 For any $\left(\theta\right.$, a) s.t. $m_{\theta, \mathbf{a}}^{\star}(\mathbf{X})$ in (10) is well-defined, $R(\theta$, a) is a monotone increasing function in $\theta>0$ for any portfolio a (and monotone decreasing for $\theta<0$ ).

Proof See "Appendix A".

Let us underline that the previous lemma shows a general property that does not depend on the distribution of $\mathbf{X}$ and on the measure of risk $V_{\mathbf{a}}(\mathbf{X})$. As already stated in the introduction, in this paper we consider $\mathbf{X}$ distributed as a multivariate normal $\mathbf{X} \sim N(\boldsymbol{\mu}, \Sigma)$ and the general mean-variance framework (i.e. with the measure of risk defined as in (5)). We now deduce a necessary and sufficient condition for which the change of measure (10) is well-defined and we find the distribution of $\mathbf{X}$ in the alternative model for any portfolio $\mathbf{a} \in \mathcal{A}$.

Lemma 2 Let $\mathbf{X} \sim N(\boldsymbol{\mu}, \Sigma)$, with $\Sigma$ a positive definite matrix with strictly positive diagonal elements, the change of measure $m_{\theta, \mathbf{a}}^{\star}(\mathbf{X})$ in (10) is well-defined if and only if $\theta \in\left[0, \theta_{\max }(\mathbf{a})\right)$ where

$$
\theta<\theta_{\max }(\mathbf{a}):=\frac{1}{\gamma \mathbf{a}^{T} \Sigma \mathbf{a}} .
$$

Moreover, for any $\mathbf{a} \in \mathcal{A}$, in the alternative model $\tilde{f}(\mathbf{X})$ corresponding to $m_{\theta, \mathbf{a}}^{\star}(\mathbf{X}), \mathbf{X}$ is distributed as a multivariate normal r.v., i.e. $\mathbf{X} \sim N(\tilde{\boldsymbol{\mu}}, \tilde{\Sigma})$, where

$$
\tilde{\boldsymbol{\mu}}=\boldsymbol{\mu}-\theta \tilde{\Sigma} \mathbf{a}, \quad \tilde{\Sigma}=\left(\Sigma^{-1}-\theta \gamma \mathbf{a} \mathbf{a}^{T}\right)^{-1} .
$$

Proof First, we prove that $m_{\theta, \mathbf{a}}^{\star}(\mathbf{X})$ in (10) is well defined. Let us observe that a necessary and sufficient condition to have a well-defined change of measure (10) is that $\mathbb{E}\left[\exp \left(\theta V_{\mathbf{a}}(\mathbf{X})\right)\right]$ is finite. We consider $\mathbf{X} \sim N(\boldsymbol{\mu}, \Sigma)$ and $V_{\mathbf{a}}(\mathbf{X})$ as in (5), thus we get

$$
\mathbb{E}\left[\exp \left(\theta V_{\mathbf{a}}(\mathbf{X})\right)\right]=\int d \mathbf{X} \frac{1}{\sqrt{(2 \pi)^{n} \operatorname{det}(\Sigma)}} \exp \left(-\theta \mathbf{a}^{T} \mathbf{X}-\frac{1}{2}(\mathbf{X}-\boldsymbol{\mu})^{T} \tilde{\Sigma}^{-1}(\mathbf{X}-\boldsymbol{\mu})\right),
$$


where $\tilde{\Sigma}^{-1}:=\Sigma^{-1}-\theta \gamma \mathbf{a a}^{T}$ is a symmetric matrix.

The integral is finite if and only if the matrix $\tilde{\Sigma}^{-1}$ is positive-definite. To prove this fact we proceed in two steps: first we compute the determinant of $\tilde{\Sigma}^{-1}$ and we then state a property on the signs of its eigenvalues.

To compute the determinant, we use the Matrix Determinant Lemma (see e.g. [7, Theorem 18.1.1, p. 416]) that states

$$
\operatorname{det}\left(\Sigma^{-1}-\theta \gamma \mathbf{a a}^{T}\right)=\left(1-\theta \gamma \mathbf{a}^{T} \Sigma \mathbf{a}\right) \operatorname{det}\left(\Sigma^{-1}\right) .
$$

Thus, the determinant of $\tilde{\Sigma}^{-1}$ is positive if and only if the condition (12) holds. If det $\tilde{\Sigma}^{-1}$ is positive, then $\tilde{\Sigma}^{-1}$ is also invertible; we define $\tilde{\Sigma}$ as its inverse. We have verified that (12) is a necessary condition to get $\tilde{\Sigma}^{-1}$, thus $\tilde{\Sigma}$, positive-definite.

We now prove that the condition $\theta<\theta_{\max }(\mathbf{a})$ is also sufficient to have the matrix $\tilde{\Sigma}^{-1}$ positive-definite. Let $\lambda_{i}$ be the eigenvalues of $\Sigma$. The eigenvalues of the inverse matrix $\Sigma^{-1}$ are the reciprocals $1 / \lambda_{i}$. Let us define $1 / \tilde{\lambda}_{i}$ the eigenvalues of $\tilde{\Sigma}^{-1}$. The following inequalities hold (see e.g. [3, Theorem 17, pp. 64-66])

$$
\frac{1}{\tilde{\lambda}_{1}} \leq \frac{1}{\lambda_{1}} \leq \frac{1}{\tilde{\lambda}_{2}} \leq \frac{1}{\lambda_{2}} \leq \cdots \leq \frac{1}{\tilde{\lambda}_{n}} \leq \frac{1}{\lambda_{n}} .
$$

Because the matrix $\Sigma^{-1}$ is positive-definite, $1 / \lambda_{i}$ are all positive, thus $\tilde{\Sigma}^{-1}$ has $n-1$ positive eigenvalues. Also having $\tilde{\Sigma}^{-1}$ a positive determinant (cf. Eq. (14)), we conclude that it is positive-definite and condition (12) is necessary and sufficient to have the whole problem well defined. In this case, after a completion of the square, we get

$$
\mathbb{E}\left[\exp \left(\theta V_{\mathbf{a}}(\mathbf{X})\right)\right]=\frac{1}{\sqrt{\operatorname{det}\left(\Sigma \tilde{\Sigma}^{-1}\right)}} \exp \left(-\theta \mathbf{a}^{T} \boldsymbol{\mu}+\frac{1}{2} \theta^{2} \mathbf{a}^{T} \tilde{\Sigma} \mathbf{a}\right) .
$$

Second, we consider $\tilde{f}(\mathbf{X})$, the density of $\mathbf{X}$ in the alternative model. For any $\mathbf{a} \in \mathcal{A}$, it is

$$
\tilde{f}(\mathbf{X})=m_{\theta, \mathbf{a}}^{\star}(\mathbf{X}) f(\mathbf{X}),
$$

which is well-defined if and only if $m_{\theta, \mathbf{a}}^{\star}(\mathbf{X})$ is well defined. In this case, $\tilde{f}(\mathbf{X})$ is a Gaussian density with mean and variance (13).

We notice that in the best-case approach, with $\theta<0$, it is not necessary to impose any additional condition for $\theta$; i.e., the alternative measure is well defined $\forall \theta \in \mathfrak{R}^{-}$: this is the only difference that should be considered when dealing with the best-case approach.

Condition (12) determines the domain with all possible values of $\theta$ that allow a well-posed problem, not limited to only small values of $\theta$ and to asymptotic results, as in [5, p. 31]. In the rest of this paper, we consider $\theta$ and $\mathbf{a}$ in the domain $\mathcal{D}$ defined as

$$
\mathcal{D}:=\left\{(\theta, \mathbf{a}) \text { s.t. } \theta \mathbf{a}^{T} \Sigma \mathbf{a}<\frac{1}{\gamma}\right\} .
$$

We now consider the two external optimisation problems in (4). First, let us define the Lagrangian function computed in the optimal change of measure

$$
\mathcal{L}(\theta, \mathbf{a}):=\mathcal{L}\left(\theta, \mathbf{a} ; m_{\theta, \mathbf{a}}^{\star}\right)=\frac{1}{\theta} \ln \mathbb{E}\left[\exp \left(\theta V_{\mathbf{a}}(\mathbf{X})\right)\right]+\frac{\eta}{\theta} .
$$

obtained substituting the optimal change of measure (10) in (4), with $V_{\mathbf{a}}(\mathbf{X})$ defined in (5). Thus, the optimisation problem to be solved becomes

$$
\inf _{\mathbf{a}} \inf _{\theta>0} \mathcal{L}(\theta, \mathbf{a}) \text {. }
$$


The standard technique to solve this problem is to exchange the order of the other two minimisation problems in (17). Before entering into details, we state some properties for the Lagrangian function in the following lemma.

Lemma $3 \operatorname{Let}(\theta, \mathbf{a}) \in \mathcal{D}$. In the alternative worst-case approach, $\mathcal{L}(\theta, \mathbf{a})$ is convex in $\mathbf{a}$ and it has a unique minimum in $\theta$, which is an interior point of the set (12); moreover, in the alternative model, the relative entropy (11) becomes

$$
R(\theta, \mathbf{a})=\frac{\theta}{2} S \Gamma(S ; \theta, \gamma)+\frac{1}{2} \ln (1-\theta \gamma S),
$$

where

$$
S:=\mathbf{a}^{T} \Sigma \mathbf{a}
$$

and

$$
\Gamma(S ; \theta, \gamma):=\frac{\gamma(1-\theta \gamma S)+\theta}{(1-\theta \gamma S)^{2}}
$$

Proof First, we prove that the Lagrangian function in the alternative worst-case approach (i.e. with $\theta>0)$ is convex in $\mathbf{a}$, s.t. $(\theta, \mathbf{a}) \in \mathcal{D}$. We can apply Sherman and Morrison [16] formula to get

$$
\tilde{\Sigma}=\Sigma+\frac{\theta \gamma \Sigma \mathbf{a a}^{T} \Sigma}{1-\theta \gamma \mathbf{a}^{T} \Sigma \mathbf{a}}
$$

then, we obtain

$$
\mathbb{E}\left[\exp \left(\theta V_{\mathbf{a}}(\mathbf{X})\right)\right]=\frac{1}{\sqrt{1-\theta \gamma \mathbf{a}^{T} \Sigma \mathbf{a}}} \exp \left(-\theta \mathbf{a}^{T} \boldsymbol{\mu}+\frac{1}{2} \theta^{2} \frac{\mathbf{a}^{T} \Sigma \mathbf{a}}{1-\theta \gamma \mathbf{a}^{T} \Sigma \mathbf{a}}\right) .
$$

The Lagrangian function in (16) becomes

$$
\mathcal{L}(\theta, \mathbf{a})=-\frac{1}{2 \theta} \ln \left(1-\theta \gamma \mathbf{a}^{T} \Sigma \mathbf{a}\right)-\mathbf{a}^{T} \boldsymbol{\mu}+\frac{1}{2} \frac{\theta \mathbf{a}^{T} \Sigma \mathbf{a}}{1-\theta \gamma \mathbf{a}^{T} \Sigma \mathbf{a}}+\frac{\eta}{\theta} .
$$

Recalling that the sum of convex functions is itself a convex function (see e.g. [1, Section 3.2.1, p. 79]), we focus on the non-linear terms in $\mathbf{a}$ in (23). We define

$$
L(\theta, S):=-\frac{1}{2 \theta} \ln (1-\theta \gamma S)+\frac{1}{2} \frac{\theta S}{1-\theta \gamma S},
$$

where $S$ is defined in (19). For $(\theta, \mathbf{a}) \in \mathcal{D}$ the function $L(\theta, S)$ is infinitely differentiable and it is increasing and convex in $S$ for $\theta>0$; moreover, $S$ is convex in a. Thus, using the composition rule for convexity (see e.g. [1, Section 3.2.4, p. 84]), we can conclude that $L\left(\theta, \mathbf{a}^{T} \Sigma \mathbf{a}\right)$ is convex in a and then the Lagrangian function (23) is convex in $\mathbf{a}$.

Second, we prove the expression (18) for $R(\theta, \mathbf{a})$. From (11), we have

$$
R(\theta, \mathbf{a})=\mathbb{E}\left[m_{\theta, \mathbf{a}}^{\star}(\mathbf{X}) \ln m_{\theta, \mathbf{a}}^{\star}(\mathbf{X})\right] .
$$

After some simplifications, we get

$$
R(\theta, \mathbf{a})=\frac{\gamma}{2} \theta \mathbb{E}\left[\left(\mathbf{a}^{T} \tilde{\mathbf{X}}\right)^{2}\right]-\theta \mathbb{E}\left[\mathbf{a}^{T} \tilde{\mathbf{X}}\right]+\ln \sqrt{\operatorname{det}\left(\Sigma \tilde{\Sigma}^{-1}\right)}-\frac{1}{2} \theta^{2} \mathbf{a}^{T} \tilde{\Sigma} \mathbf{a}
$$

where $\tilde{\mathbf{X}}$ is a Gaussian r.v. with mean $-\theta \tilde{\Sigma}$ a and variance in (13). Finally, using Eq. (21) and substituting (19) and (20), the relative entropy (18) follows. 
Finally, we prove that the Lagrangian function for any given a has a unique minimum in $\theta$, called $\tilde{\theta}$, and, in particular, that it is a monotone decreasing function in $(-\infty, \tilde{\theta})$ and a monotone increasing function in $\left(\tilde{\theta}, \theta_{\max }(\mathbf{a})\right)$.

Because $m_{\theta, \mathbf{a}}^{\star}$ is optimal for the Lagrangian function in (4) and the alternative model $\tilde{f}(\mathbf{X})$ is a sufficiently regular function (a multinomial Gaussian p.d.f.), we can exchange the derivative with the expected value (see e.g. [15, Th. 4, p. 429]) and we get

$\frac{\partial \mathcal{L}(\theta, \mathbf{a})}{\partial \theta}=\frac{\partial}{\partial \theta}\left\{\mathbb{E}\left[m_{\theta, \mathbf{a}}^{\star}(\mathbf{X}) V_{\mathbf{a}}(\mathbf{X})-\frac{1}{\theta}\left(m_{\theta, \mathbf{a}}^{\star}(\mathbf{X}) \ln m_{\theta, \mathbf{a}}^{\star}(\mathbf{X})-\eta\right)\right]\right\}=\frac{1}{\theta^{2}}(R(\theta, \mathbf{a})-\eta)$.

Observing that the relative entropy $R(\theta, \mathbf{a})$ is null in $\theta=0 \forall \mathbf{a}$, it is monotone increasing for $\theta>0$ (cf. Lemma 1) and it tends to infinity if $\theta \rightarrow \theta_{\max }(\mathbf{a})$ (straightforward using (18) and (20)), the thesis follows.

Glasserman and $\mathrm{Xu}$ [5] prove that, under certain conditions, it is possible to exchange the two infima in (17) and subsequently they solve the optimisation problem in the variables a and $\theta$ numerically. We use the same inversion as Glasserman and $\mathrm{Xu}$ [5], but we solve analytically the problem: as already stated in the introduction, this is our main theoretical contribution.

To find the optimal portfolio in the alternative measure and the corresponding optimal change of measure, we recall Proposition 2.1 of Glasserman and $\mathrm{Xu}$ [5] for the portfolio selection.

Lemma 4 Let $(\theta, \mathbf{a}) \in \mathcal{D}$. The optimisation problem (17) is equivalent to

$$
\inf _{\theta>0} \inf _{\mathbf{a} \in \mathcal{A}} \mathcal{L}(\theta, \mathbf{a})
$$

where $\mathcal{L}(\theta, \mathbf{a})$ is defined in (16); the optimal portfolio $\mathbf{a}^{\star}(\theta)$ in the alternative measure is

$$
\mathbf{a}^{\star}(\theta)=\arg \inf _{\mathbf{a} \in \mathcal{A}} \frac{1}{\theta} \ln \mathbb{E}\left[\exp \left(\theta V_{\mathbf{a}}(\mathbf{X})\right)\right]
$$

and the worst-case change of measure is

$$
m_{\theta}^{\star}(\mathbf{X})=\frac{\exp \left(\theta V_{\mathbf{a}^{\star}(\theta)}(\mathbf{X})\right)}{\mathbb{E}\left[\exp \left(\theta V_{\mathbf{a}^{\star}(\theta)}(\mathbf{X})\right)\right]} .
$$

Proof See Proposition 2.1 in Glasserman and $\mathrm{Xu}$ [5], applied to the mean-variance case.

This lemma is an important result known from literature about the worst-case model risk. As in Glasserman and $\mathrm{Xu}$ [5], we follow the same approach in this paper. We first find the optimal worst-case portfolio a given $\theta>0$ and then select the optimal value of $\theta$ within the KL-ball $P_{\eta}$. It is straightforward to prove that in the best-case approach the same equations of Lemma 4 hold with $\theta<0$.

In the rest of this paper, we find an analytical solution for (25) in two cases: the general mean-variance framework in Sect. 3 and the special case considered by Glasserman and Xu [5] with a constraint on the mean in the alternative model, analysed in Sect 4.

\section{Analytical solution for the worst-case portfolio selection}

In this section, we solve analytically the optimisation problem (26) and find the optimal portfolio in the alternative model for a given value of $\theta>0$; i.e., the robust portfolio in the 
Fig. 1 Plot of $S$ as a function of $1 / \Gamma$ in Eq. (29) (continuous black line) and $1 / \Gamma$ as a function of $S$ in Eq. (20) for $S \in[1 / C, 1 / \theta \gamma)$ (dot-dashed red line). The system of two equations admits a unique solution. (Color figure online)

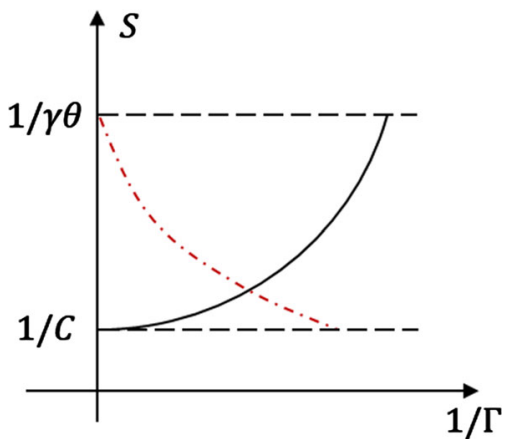

mean-variance framework. Then, we prove that the optimum $\theta$ in (25) stays on the surface of the ball $P_{\eta}$.

Theorem 5 Let $(\theta, \mathbf{a}) \in \mathcal{D}$. In the alternative worst-case approach, the optimal portfolio $\mathbf{a}^{\star}(\theta)$ is

$$
\mathbf{a}^{\star}(\theta)=\frac{A}{\Gamma\left(S^{\star}(\theta) ; \theta, \gamma\right)} \frac{\Sigma^{-1} \boldsymbol{\mu}}{A}+\left(1-\frac{A}{\Gamma\left(S^{\star}(\theta) ; \theta, \gamma\right)}\right) \frac{\Sigma^{-1} \mathbf{1}}{C}
$$

and the quantity $S^{\star}(\theta)$ is the unique solution of

$$
S=\frac{1}{C}\left(\frac{D}{\Gamma(S ; \theta, \gamma)^{2}}+1\right)
$$

where $\Gamma(S ; \theta, \gamma)$ is defined in (20). The constants A, C, D have been defined in (7).

Proof In the alternative worst-case approach, it is possible to find the optimal portfolio solving (26). Using (22) and introducing a Lagrange multiplier for the constraint $\mathbf{a} \in \mathcal{A}$ (i.e. $\left.\mathbf{a}^{T} \mathbf{1}=1\right)$, (26) is equivalent to

$$
\arg \inf _{\mathbf{a}}-\frac{1}{2 \theta} \ln \left(1-\theta \gamma \mathbf{a}^{T} \Sigma \mathbf{a}\right)-\mathbf{a}^{T} \boldsymbol{\mu}+\frac{1}{2} \theta \frac{\mathbf{a}^{T} \Sigma \mathbf{a}}{1-\theta \gamma \mathbf{a}^{T} \Sigma \mathbf{a}}+\alpha\left(1-\mathbf{a}^{T} \mathbf{1}\right) .
$$

Imposing the first order condition on a, we get the Eq. (28) for the optimal a, where $S$ is defined in (19). By substituting (28) in (19), we get the Eq. (29) for the optimal $S$.

Let us study the solutions of Eq. (29) given $\Gamma(S ; \theta, \gamma)$ in (20). The domain of $S$ is s.t. $S$ must be included in the interval

$$
\frac{1}{C} \leq S<\frac{1}{\theta \gamma},
$$

because i) $S=1 / C$ corresponds to the minimum-variance case even in the alternative model and ii) $(\theta, \mathbf{a}) \in \mathcal{D}$, i.e. $\theta S<1 / \gamma$.

First, let us consider $S$ as a function of $1 / \Gamma$ in Eq. (29) in the domain (30), which is one branch of a parabola (and then monotone increasing) with a minimum in $(0,1 / C)$. Then, consider $1 / \Gamma$ as a function of $S$ in Eq. (20) in the same domain for $S$, which is equal to a positive value in $S=1 / C$ and it is derivable in the domain of $S$ with a derivative always strictly negative and it tend to zero in the limit $S \rightarrow 1 /(\theta \gamma)$. Hence, Eq. (29) has a unique solution, as shown in Fig. 1. 
Depending on $\theta$, the worst-case mean-variance optimal portfolio is the (unique) analytical solution in Eq. (29). Let us observe that, in the mean-variance framework, the optimal worstcase portfolio is similar to the optimal nominal one (8). Also in the worst-case approach a two mutual fund theorem holds: the optimal portfolio is the linear combination-with a different weight—of the same two portfolios $\mathbf{a}_{1}^{\star}$ and $\mathbf{a}_{0}^{\star}$ of the nominal problem. The solution in the alternative model has exactly the same form of the nominal one with an increased risk aversion "parameter" $\Gamma$ in the worst-case approach $(\Gamma>\gamma$ for $\theta>0)$. It can be shown that a similar solution holds also in the best-case approach with a decreased risk aversion $(\Gamma<\gamma$ for $\theta<0$ ).

Let us notice that, given the optimal portfolio $\mathbf{a}^{\star}(\theta)$, we are now able to find the corresponding relative entropy, i.e. the KL-divergence between the nominal and the alternative model, depending only on $\theta$. Substituting the unique solution of (29) in (18), we get

$$
R(\theta):=R\left(\theta, \mathbf{a}^{\star}(\theta)\right)=\frac{\theta}{2} S^{\star}(\theta) \Gamma\left(S^{\star}(\theta) ; \theta, \gamma\right)+\frac{1}{2} \ln \left(1-\theta \gamma S^{\star}(\theta)\right) .
$$

We conclude this section proving that the optimal $\theta$ in the optimisation problem (25) stays on the surface of the ball $P_{\eta}$. This result is crucial because it solves completely (25), i.e. the mean-variance portfolio selection in the worst-case approach.

Proposition 6 Let $\left(\theta, \mathbf{a}^{\star}(\theta)\right) \in \mathcal{D}$. The optimal value $\theta^{\star}$ for $\theta$ in optimisation problem (25) is on the surface of the ball $P_{\eta}$, i.e. $R\left(\theta^{\star}\right)=\eta$ where $R(\theta)$ is defined in (31).

Proof We first show that the optimum $\theta$ that solves the original optimisation problem (4) is on the surface of the ball $P_{\eta}$ for any given portfolio a; then, we consider problem (25) obtained inverting the two infima.

First, we consider the internal maximisation problem in (3) for a given a, that is the primal problem. Following the notation in [1, pp. 216-225] we call the primal problem $p$, we indicate with $d$ the corresponding Lagrangian dual problem in (4), while $p^{\star}$ and $d^{\star}$ denote the optimal values respectively of the primal problem and of the dual one.

Because the objective function is convex in $m$ and the set $\{m: \mathbb{E}[m(\mathbf{X}) \ln m(\mathbf{X})]<\eta\}$ is non-empty, Slater's theorem ensures that strong duality holds, i.e. $p^{\star}=d^{\star}$ (see e.g. [1, Section 5.2.3, p. 226]). In other words, for a given $\mathbf{a}$, given $m_{\mathbf{a}}^{\star p}(\mathbf{X})$ a primal optimum and $\left\{\tilde{\theta}, m_{\tilde{\theta}, \mathbf{a}}^{\star}(\mathbf{X})\right\}$ a dual optimum where $\tilde{\theta}$ is a function of $\mathbf{a}$, we have

$$
\begin{aligned}
p^{\star} & =d^{\star}=\inf _{\theta>0} \sup _{m} \mathbb{E}\left[m(\mathbf{X}) V_{\mathbf{a}}(\mathbf{X})-\frac{1}{\theta}(m(\mathbf{X}) \ln m(\mathbf{X})-\eta)\right]= \\
& =\sup _{m} \inf _{\theta>0} \mathbb{E}\left[m(\mathbf{X}) V_{\mathbf{a}}(\mathbf{X})-\frac{1}{\theta}(m(\mathbf{X}) \ln m(\mathbf{X})-\eta)\right]= \\
& =\sup _{m} \mathbb{E}\left[m(\mathbf{X}) V_{\mathbf{a}}(\mathbf{X})-\frac{1}{\tilde{\theta}}(m(\mathbf{X}) \ln m(\mathbf{X})-\eta)\right] \geq \\
& \geq \mathbb{E}\left[m_{\mathbf{a}}^{\star p}(\mathbf{X}) V_{\mathbf{a}}(\mathbf{X})-\frac{1}{\tilde{\theta}}\left(m_{\mathbf{a}}^{\star p}(\mathbf{X}) \ln m_{\mathbf{a}}^{\star p}(\mathbf{X})-\eta\right)\right] \geq \\
& \geq \mathbb{E}\left[m_{\mathbf{a}}^{\star p}(\mathbf{X}) V_{\mathbf{a}}(\mathbf{X})\right]=p^{\star} .
\end{aligned}
$$

The second line comes from [1, Eq. (5.45), p. 238]. The third line considers the optimum $\tilde{\theta}$ of the dual problem. The fourth line follows because the supremum of the Lagrangian over $m$ is greater or equal to its value at any other $m(\mathbf{X})$ and then also choosing $m(\mathbf{X})=m_{\mathbf{a}}^{\star p}(\mathbf{X})$. The last inequality is due to the fact that the second term in fourth line is non-negative. 
Thus, since all inequalities hold with equality, we can draw two conclusions. First, $m_{\mathbf{a}}^{\star p}(\mathbf{X})$ maximises the Lagrangian. This result, added to the concavity of the Lagrangian in $m$, implies that $m_{\mathbf{a}}^{\star p}(\mathbf{X})=m_{\tilde{\theta}, \mathbf{a}}^{\star}(\mathbf{X})$.

Second, the following equality holds

$$
\frac{1}{\tilde{\theta}} \mathbb{E}\left[\left(m_{\tilde{\theta}, \mathbf{a}}^{\star}(\mathbf{X}) \ln m_{\tilde{\theta}, \mathbf{a}}^{\star}(\mathbf{X})-\eta\right)\right]=0 .
$$

Because $\theta$ must remain finite, due to condition (12), we get that the expectation in (32) must be null; i.e., $\tilde{\theta}$ stays on the surface of the ball $P_{\eta}$, or equivalently $R(\tilde{\theta}$, a) $=\eta \forall \mathbf{a}$ s.t. $(\tilde{\theta}, \mathbf{a}) \in \mathcal{D}$.

Because (i) the relative entropy $R(\theta, \mathbf{a})$ is a monotone increasing function in $\theta$ (cf. Lemma 1), (ii) it is null when $\theta=0$ and (iii) it tends to infinity in the limit $\theta \rightarrow \theta_{\max }(\mathbf{a})$ (cf. also the end in the proof of Lemma 3), then there exists a unique solution of (32) for

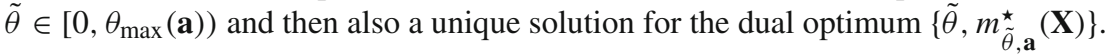

In Lemma 4 we have proven that it is possible to change the order of the two infima in (4), obtaining the same solution. This is the inverted dual problem (25). Thus, the optimum value $\theta^{\star}$ of the inverted dual problem is as well on the surface of the ball, that ends the proof

The result proved in Proposition 6 allows us (i) to avoid the numerical optimisation in $\theta$ and (ii) to identify the optimal $\theta$, corresponding to the divergence $\eta$, as the (unique) positive value on the surface of the ball $P_{\eta}$. This completely solves the problem (25).

\section{Mean-variance with constant mean}

In this section we analyse in detail the mean-variance case considered by Glasserman and Xu [5], in which they consider a special case with a constraint on the mean vector that must be equal to the nominal mean $\boldsymbol{\mu}$ even in the alternative model. The problem in this case becomes

$$
\left\{\begin{array}{l}
\inf _{\mathbf{a} \in \mathcal{A}} \sup _{m \in P_{\eta}} \mathbb{E}\left[m(\mathbf{X})\left(\frac{\gamma}{2} \mathbf{a}^{T}(\mathbf{X}-\boldsymbol{\mu})(\mathbf{X}-\boldsymbol{\mu})^{T} \mathbf{a}-\mathbf{a}^{T} \mathbf{X}\right)\right] \\
\text { s.t. } \mathbb{E}[m(\mathbf{X}) \mathbf{X}]=\boldsymbol{\mu}
\end{array}\right.
$$

as in $[5$, p. 36].

In the remaining part of this section, first we show-in the next lemma-that the constrained problem (33) can be reduced to an unconstrained one with a different measure of risk $V_{\mathbf{a}}^{G X}(\mathbf{X})$, then we replicate in this special case all results proven in previous sections.

Lemma 7 Let $\mathbf{X} \sim N(\boldsymbol{\mu}, \Sigma)$, with $\Sigma$ a positive definite matrix with strictly positive diagonal elements, the mean-variance problem with constant mean (33) is equivalent to

$$
\inf _{\mathbf{a} \in \mathcal{A}} \sup _{m \in P_{\eta}} \mathbb{E}\left[m(\mathbf{X}) V_{\mathbf{a}}^{G X}(\mathbf{X})\right]
$$

with $V_{\mathbf{a}}^{G X}(\mathbf{X})$ the measure of risk defined in (9).

Proof See "Appendix A".

We can then replicate in this case the results in Sects. 2 and 3. First, let us show that the basic properties of Lemmas 2, 3 and 4 can be adapted to this special case, considering the measure of risk (9). This is proven in the next Lemmas 8 and 9. 
Lemma 8 Let $(\theta, \mathbf{a}) \in \mathcal{D}$. The change of measure $m_{\theta, \mathbf{a}}^{\star}(\mathbf{X})$ in $(10)$ is well-defined if and only if condition (12) holds. Moreover, for any $\mathbf{a} \in \mathcal{A}$, in the alternative model $\tilde{f}(\mathbf{X})$ corresponding to $m_{\theta, \mathbf{a}}^{\star}(\mathbf{X}), \mathbf{X}$ is distributed as a multivariate normal r.v., i.e. $\mathbf{X} \sim N(\boldsymbol{\mu}, \tilde{\Sigma})$, with $\tilde{\Sigma}$ as in (13).

Proof See proof of Lemma 2, noting that $\mathbb{E}\left[\exp \left(\theta V_{\mathbf{a}}^{G X}(\mathbf{X})\right)\right]$ attains the same value as in mean-variance framework with a linear constant term in the exponential $-\theta \mathbf{a}^{T} \boldsymbol{\mu}$ instead of the linear stochastic term $-\theta \mathbf{a}^{T} \mathbf{X}$. Thus, the first part of the proof holds and it is straightforward to show that $\tilde{f}(\mathbf{X})$ is the density of a Gaussian r.v. with mean $\boldsymbol{\mu}$ (the new constraint that we are imposing) and variance $\tilde{\Sigma}$

Defining $\mathcal{L}^{G X}(\theta, \mathbf{a})$ as in (16), using $V_{\mathbf{a}}^{G X}(\mathbf{X})$ instead of $V_{\mathbf{a}}(\mathbf{X})$, we can show that results similar to Lemmas 3 and 4 hold as well.

Lemma $9 \operatorname{Let}(\theta, \mathbf{a}) \in \mathcal{D} \cdot \mathcal{L}^{G X}(\theta, \mathbf{a})$ is convex in $\mathbf{a}$ and it has a unique minimum in $\theta$, interior point of the set (12). In the alternative worst-case approach, the relative entropy is

$$
R(\theta, \mathbf{a})=\frac{\theta}{2} S \Gamma^{G X}(S ; \theta, \gamma)+\frac{1}{2} \ln (1-\theta \gamma S),
$$

where

$$
\Gamma^{G X}(S ; \theta, \gamma):=\frac{\gamma}{1-\theta \gamma S}
$$

Moreover, it is possible to exchange the order of the two inferior in the optimisation problem (4), that becomes equivalent to

$$
\inf _{\theta>0} \inf _{\mathbf{a} \in \mathcal{A}} \mathcal{L}^{G X}(\theta, \mathbf{a})
$$

the optimal portfolio in the alternative measure is found solving

$$
\mathbf{a}^{\star}(\theta)=\arg \inf _{\mathbf{a} \in \mathcal{A}} \frac{1}{\theta} \ln \mathbb{E}\left[\exp \left(\theta V_{\mathbf{a}}^{G X}(\mathbf{X})\right)\right] .
$$

Proof See proof of Lemmas 3 and 4, noting that

$$
\mathcal{L}^{G X}(\theta, \mathbf{a})=-\frac{1}{2 \theta} \ln (1-\theta \gamma S)-\mathbf{a}^{T} \boldsymbol{\mu}+\frac{\eta}{\theta}
$$

and for the relative entropy that

$$
R(\theta, \mathbf{a})=\mathbb{E}\left[m_{\theta}^{\star}(\mathbf{X}) \frac{\theta \gamma}{2} \mathbf{a}^{T}(\mathbf{X}-\boldsymbol{\mu})(\mathbf{X}-\boldsymbol{\mu})^{T} \mathbf{a}\right]-\ln \mathbb{E}\left[\exp \left(\frac{\theta \gamma}{2} \mathbf{a}^{T}(\mathbf{X}-\boldsymbol{\mu})(\mathbf{X}-\boldsymbol{\mu})^{T} \mathbf{a}\right)\right] .
$$

Following similar computations as in mean-variance case, all previous results hold.

Then, similarly to Theorem 5 we can find the closed form solution of problem (37) for a given positive $\theta$ and prove that the optimum stays on the surface of the ball $P_{\eta}$ also in this special case.

Theorem 10 Let $(\theta, \mathbf{a}) \in \mathcal{D}$. In the alternative worst-case approach, the optimal portfolio is

$$
\mathbf{a}^{\star}(\theta)=\frac{A}{\Gamma^{G X}(\theta, \gamma)} \frac{\Sigma^{-1} \boldsymbol{\mu}}{A}+\left(1-\frac{A}{\Gamma^{G X}(\theta, \gamma)}\right) \frac{\Sigma^{-1} \mathbf{1}}{C}
$$


where

$$
\Gamma^{G X}(\theta, \gamma)=\frac{\gamma C+\sqrt{\gamma^{2} C^{2}+4 \theta \gamma(C-\theta \gamma) D}}{2(C-\theta \gamma)},
$$

with $\Gamma^{G X}(\theta, \gamma)>0$.

Proof As in the mean-variance case, it is possible to find the optimal portfolio in the alternative measure solving (37), that becomes

$$
\inf _{\mathbf{a}: \mathbf{a}^{T} \mathbf{1}=1} \frac{1}{\theta} \ln \mathbb{E}\left[\exp \left(\frac{\theta \gamma}{2} \mathbf{a}^{T}(\mathbf{X}-\boldsymbol{\mu})(\mathbf{X}-\boldsymbol{\mu})^{T} \mathbf{a}\right)\right]-\mathbf{a}^{T} \boldsymbol{\mu}+\frac{\eta}{\theta}
$$

that, introducing a Lagrange multiplier for the constraint $\mathbf{a}^{T} \mathbf{1}=1$, is equivalent to

$$
\inf _{\mathbf{a}}-\frac{1}{2 \theta} \ln \left(1-\theta \gamma \mathbf{a}^{T} \Sigma \mathbf{a}\right)-\mathbf{a}^{T} \boldsymbol{\mu}+\frac{\eta}{\theta}-\lambda\left(\mathbf{a}^{T} \mathbf{1}-1\right) .
$$

Following the same method as in Theorem 5, we get Eq. (28) with $\Gamma^{G X}(S ; \theta, \gamma)$ defined in (36) instead of $\Gamma(S ; \theta, \gamma)$ defined in (20).

Moreover, as in Theorem 5, by substituting (28) in (19), we get the Eq. (29). In this special case, Eqs. (29) and (36) represent a second order degree system, that can be solved in closed form. We obtain the following solution for a second order degree equation in the variable $\Gamma^{G X}$

$$
\Gamma^{G X}(\theta, \gamma)=\frac{\gamma C \pm \sqrt{\gamma^{2} C^{2}+4 \theta \gamma(C-\theta \gamma) D}}{2(C-\theta \gamma)} .
$$

As in the mean-variance case, the domain for $S$ is (30), and then $C>\theta \gamma$. Because from (36) we must have that $\Gamma^{G X}(S ; \theta, \gamma)>0$, we can deduce that the solution for $\Gamma^{G X}(\theta, \gamma)$ with the negative sign is non-acceptable in the worst-case approach, thus (39) is the unique solution for $\Gamma^{G X}(\theta, \gamma)$, that concludes the proof

As in the mean-variance case, let us notice that, given the optimal portfolio (38), we are able to obtain from (35) the corresponding relative entropy that depends only on $\theta$; i.e.,

$$
R(\theta)=\frac{1}{2}\left(\frac{\Gamma^{G X}(\theta, \gamma)}{\gamma}-1-\ln \frac{\Gamma^{G X}(\theta, \gamma)}{\gamma}\right) .
$$

Also Proposition 6 can be replicated in this special case.

Proposition 11 Let $\left(\theta, \mathbf{a}^{\star}(\theta)\right) \in \mathcal{D}$. The optimal value $\theta^{\star}$ for $\theta$ in the worst-case portfolio selection is on the ball, i.e. $R\left(\theta^{\star}\right)=\eta$, with $R(\theta)$ in (40).

Proof The proof follows the same steps of Proposition 6 with the Lagrangian and entropy of this special case.

We conclude the section noting that the value of the risk measure in this framework simply becomes

$$
\mathbb{E}\left[m_{\theta}^{\star}(\mathbf{X}) V_{\mathbf{a}^{\star}(\theta)}^{G X}(\mathbf{X})\right]=\frac{\gamma}{2} \mathbf{a}^{\star}(\theta)^{T} \tilde{\Sigma} \mathbf{a}^{\star}(\theta)-\mathbf{a}^{\star}(\theta)^{T} \boldsymbol{\mu}=\frac{1}{2 C}\left(\Gamma^{G X}(\theta, \gamma)-\frac{D}{\Gamma^{G X}(\theta, \gamma)}\right)-\frac{A}{C}
$$

where in the second equality we have used (21) and the optimal portfolio $\mathbf{a}^{\star}(\theta)$ in (38). 
In practice, an efficient way to obtain a graphical representation of the result is the following. First, identify the alternative model through the parameter $\theta$, (e.g. in a range $[0, \hat{\theta}]$ ), and then, compute the relative entropy (40) for that value of $\theta$. The numerical examples in next section are carried out in this way, obtaining at the same time the relative entropy (40) and the associated value of the risk measure (41).

\section{Equality of optimal portfolio in alternative and nominal models and numerical examples}

In this section, we analyse the cases where the optimal portfolio in the nominal model and in the alternative one are equal: this result is straightforward to achieve having deduced the analytical solution for the optimal portfolio. We prove that this equality holds only in two relevant cases: the minimum-variance problem in which the uncertainty is limited only to the covariance matrix and a symmetric case where all assets have the same mean, i.e. $\boldsymbol{\mu}=\mu \mathbf{1}$.

The minimum-variance case is an interesting subcase of the mean-variance portfolio selection - as of the mean-variance with fixed mean portfolio selection considered in Glasserman and $\mathrm{Xu}$ [5] - and it is a purely risk-based approach to portfolio construction. This corresponds to selecting a very large risk adversion parameter $\gamma$ in (5) or equivalently in (9). In this case, the measure of risk is

$$
V_{\mathbf{a}}(\mathbf{X}):=\frac{1}{2} \mathbf{a}^{T}(\mathbf{X}-\boldsymbol{\mu})(\mathbf{X}-\boldsymbol{\mu})^{T} \mathbf{a} .
$$

In the minimum-variance case, we can prove the interesting analytical result that the optimal portfolio in the worst-case approach is exactly the same as the optimal portfolio in the nominal model. Moreover, we can adapt all results obtained in previous sections to this case.

First, we can adapt Lemma 8 and Proposition 11 to the minimum-variance case, obtaining that: (i) in the alternative model, $\mathbf{X}$ is distributed as a multivariate normal r.v. with the same mean $\boldsymbol{\mu}$ as the nominal model and variance $\tilde{\Sigma}$ as in (13), if and only if the same condition (12), with $\gamma=1$, holds; (ii) the relative entropy $R(\theta)$ associated to the optimal worst-case and nominal portfolio has the same expression (40) as in previous section, with $\gamma=1$, and the optimal $\theta$ is on the surface of the ball $P_{\eta}$.

Then, we can prove that the optimal portfolio in the alternative model (i.e. the robust portfolio) is the same of the optimal one in the nominal model, as shown in the next proposition.

Proposition 12 Let $(\theta, \mathbf{a}) \in \mathcal{D}$. In the minimum-variance case, the optimisation problem (26) is equivalent to

$$
\mathbf{a}^{\star}(\theta)=\arg \inf _{\mathbf{a} \in \mathcal{A}} \mathbf{a}^{T} \Sigma \mathbf{a}
$$

thus, the optimal portfolio in the alternative model $\tilde{f}(\mathbf{X})$ is the same as the one of the nominal model $f(\mathbf{X})$, i.e. $\mathbf{a}^{\star}(\theta)=\mathbf{a}_{0}^{\star}$.

Proof The optimal portfolio is found solving optimisation problem (26). After some computations and using (14), we get

$$
\mathbb{E}\left[\exp \left(\theta V_{\mathbf{a}}(\mathbf{X})\right)\right]=\frac{1}{\sqrt{\operatorname{det}\left(\Sigma \tilde{\Sigma}^{-1}\right)}}=\frac{1}{\sqrt{1-\theta \mathbf{a}^{T} \Sigma \mathbf{a}}} .
$$


Hence, the worst-case problem (26) is equivalent to the classical problem. Then, the solution is the same of the nominal model and it is unique.

Let us stress that two main properties hold in the minimum-variance framework: not only the exponential change of measure stays in the family of multivariate normal distributions, but also the robust portfolio is equal to the optimal one in the nominal model. These two properties lead to the consequence that in the minimal-variance framework the worst-case approach corresponds to a change in the parameters of the Gaussian distribution: in this situation model risk can be explained simply as an estimation risk.

In the remaining part of this section, we get back to the mean-variance framework and we focus our attention on a symmetric case

$$
\boldsymbol{\mu}=\mu \mathbf{1}=\left[\begin{array}{c}
\mu \\
\mu \\
\vdots \\
\mu
\end{array}\right] .
$$

We first prove a general result of a necessary and sufficient condition for the equality of the optimal portfolio in the nominal and in the alternative model: in this case model risk is equivalent to an estimation risk. Then, we show some numerical examples.

In the general mean-variance framework (and also in the special case), it is possible to prove the following proposition, that guarantees the equality of the optimal portfolios in the nominal and in the alternative model.

Proposition 13 Let $(\theta, \mathbf{a}) \in \mathcal{D}$ and $\gamma$ a finite risk aversion. The optimal mean-variance portfolio in the nominal and alternative models are equal if and only if $\boldsymbol{\mu}=\mu \mathbf{1}$ with $\mu \in \mathbb{R}$.

Proof From Eqs. (8) and (28), we have

$$
\mathbf{a}^{\star}(\theta)=\mathbf{a}_{\text {nom }}^{\star} \Leftrightarrow\left(\Sigma^{-1} \boldsymbol{\mu}-\frac{A}{C} \Sigma^{-1} \mathbf{1}\right)\left(\frac{1}{\gamma}-\frac{1}{\Gamma(S, \theta, \gamma)}\right)=\mathbf{0},
$$

where $A$ and $C$ have been defined in (7) and $\mathbf{0}$ is the null vector in $\mathbb{R}^{n}$. The equation has a solution only if either i) $C \Sigma^{-1} \boldsymbol{\mu}=A \Sigma^{-1} \mathbf{1}$ or ii) $\Gamma(S, \theta, \gamma)^{-1}=\gamma^{-1} \Leftrightarrow \theta=$ 0 . While, for finite $\gamma>0$, the condition ii) cannot be fulfilled in the alternative model, condition i) proves the proposition: it corresponds to have $\boldsymbol{\mu}$ in the same direction of $\mathbf{1}$ with $\mu=A / C$.

It can be interesting to underline that Proposition 13 holds even in the mean-variance case with a constraint on the mean vector considered by Glasserman and $\mathrm{Xu}$ [5].

In order to show some numerical examples, let us consider, as in Glasserman and $\mathrm{Xu}$ [5], the case of mean-variance with fixed mean and a fully symmetric variance, i.e.

$$
\Sigma=\sigma^{2}\left[\begin{array}{cccc}
1 & \rho & \ldots & \rho \\
\rho & 1 & \ldots & \rho \\
\vdots & \vdots & \ddots & \vdots \\
\rho & \rho & \ldots & 1
\end{array}\right],
$$

with $\rho>-1 /(n-1)$. This case presents the advantage of a complete detailed analytical solution for a generic $n$ and it allows to understand in an interesting example what can 

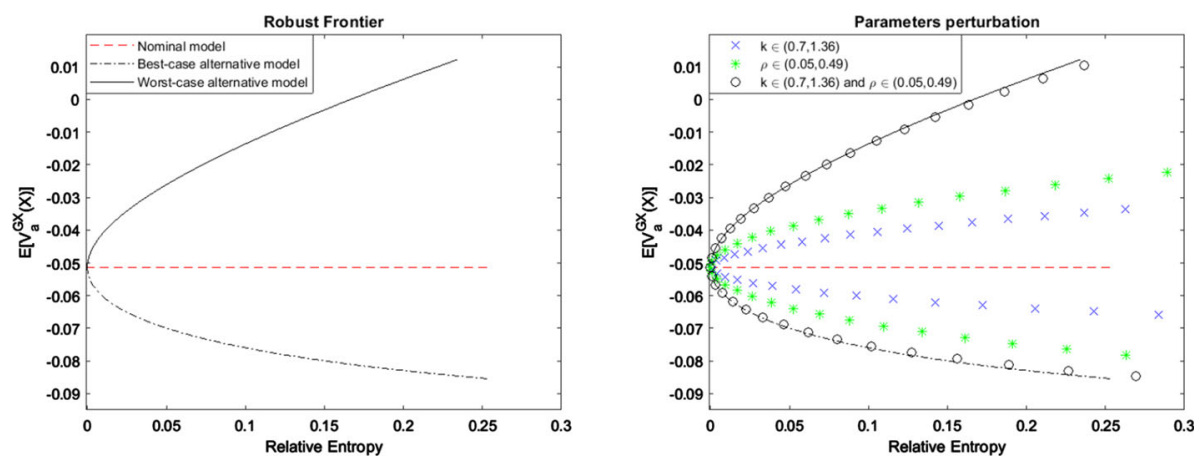

Fig. 2 Value of the risk measure versus relative entropy in the symmetric case. We consider $\gamma=1, n=10$ assets, $\mu_{i}=0.1, \Sigma_{i i}=0.3 \forall i=1, \ldots, 10$ and $\rho=0.25$. The left-hand figure shows the value of the risk measure, corresponding to the optimal portfolio, in the nominal (dashed red line) and in the alternative model (black line). Considering the maximum relative entropy $\eta \in[0,0.25]$ as in [5, Figure 1, p. 37], we compute, for each fixed value of $\eta$, the corresponding positive and negative values of $\theta^{\star}$ using (40), with which we get the value of the risk measure (41). In particular, negative $\theta^{\star}$ values correspond to the best-case alternative model (dotted-dashed line), while positive $\theta^{\star}$ values correspond to the worst-case approach (continuous line). The right-hand figure shows the value of the risk measure in the nominal model varying just the correlation parameter $\rho$ with $\rho \in[0.05,0.45]$ (green stars) and a multiplicative parameter $k$ of the variance parameter $\sigma^{2}$ with $k \in[0.72,1.32]$ (blue crosses) as in Glasserman and $\mathrm{Xu}$ [5]. We notice that the value of the risk measure obtained varying both the parameters (black circles) is the same as that obtained in the alternative model (black line). Model risk in this case reduces simply to estimation risk. (Color figure online)

happen in the numerical determination of the optimal solution in the alternative model. The eigenvalues of the variance-covariance matrix $\Sigma$ are (see e.g. Lemma 14 in "Appendix A"):

$$
\left\{\begin{array}{l}
\lambda_{1}=\sigma^{2}(1+(n-1) \rho) \text { with multiplicity } 1 \text { and eigenvector with constant weights, } \\
\lambda_{2}=\sigma^{2}(1-\rho) \quad \text { with multiplicity } n-1
\end{array}\right.
$$

Similarly, also the inverse matrix $\Sigma^{-1}$, can be computed $\forall n$.

As a first numerical example, let us consider a symmetric case $\boldsymbol{\mu}=\mu \mathbf{1}$ as considered by Glasserman and $\mathrm{Xu}$ [5] in their numerical example. In this case, the optimal portfolio in the nominal model and in the alternative model is the equally weighted one $\mathbf{a}_{0}^{\star}=1 / n$. We also have an explicit expression for condition (12) for the optimal portfolio in the alternative model $\mathbf{a}_{0}^{\star}$. Using (43), it becomes

$$
\theta<\theta_{\max }\left(\mathbf{a}_{0}^{\star}\right)=\frac{n}{\sigma^{2}(1+(n-1) \rho)}=\frac{n}{\lambda_{1}} .
$$

We consider exactly the same numerical example as that in [5, pp. 36-37] with $\gamma=1$, $n=10$ assets, $\mu_{i}=0.1, \Sigma_{i i}=\sigma^{2}=0.3 \forall i=1, \ldots, 10$ and $\rho=0.25$ and we recall that they consider the special case with a mean-variance with fixed mean. We plot the value of the risk measure for the optimal portfolio as a function of the maximum allowed relative entropy $\eta$ (cf. Fig. 1 in [5, p. 37]). The left-hand plot in Fig. 2 shows, for a set of maximum relative entropy values $\eta \in[0,0.25]$, the value of the risk measure in the alternative model in the worst-case approach (continuous black line) and in the best-case approach (dot-dashed black line).

As already stated, in this case, due to Proposition 13, the optimal portfolios in the nominal and in the alternative model coincide. This is different from the result shown in [5, Figure 1, p. 37] in which these two optimal portfolios do not coincide. This incoherence 


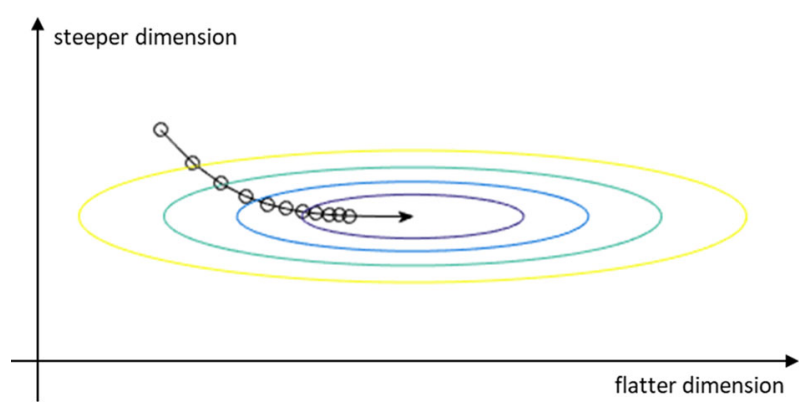

Fig. 3 Evolution of a gradient descent algorithm in the minimisation of a quadratic function in two dimensions. Similar results hold for other first order optimisation algorithms. We notice that the algorithm is slow in reaching the correct optimum in the flatter dimension. In more dimensions, this behaviour is amplified and the algorithm could not reach the optimum with a reasonable precision

might be due to a slow convergence of the numerical algorithm. We return to this point in the following.

Moreover, Glasserman and $\mathrm{Xu}$ [5] claim that "model error does not correspond to a straightforward error in parameters" (cf. p. 37). To illustrate this idea-because in the meanvariance with fixed mean framework the alternative model differs from the nominal model just for the variance matrix - these authors studied the value of the risk measure obtained by varying just two parameters: the common correlation parameter $\rho$ and a parameter $k$ that multiplies the variance parameter $\sigma^{2}$ in the the variance matrix $\Sigma$. In particular, they let the correlation parameter vary between $\rho=0.05$ and $\rho=0.45$, and the parameter $k$ vary between $k=0.72$ and $k=1.32$.

The result obtained by varying $k$ and $\rho$ separately is shown in [5, Figure 1, p. 37] and it is in agreement with blue crosses and green stars in the right-hand panel of Fig. 2. The new result is that the perturbation of both parameters $\rho$ and $k$, in the same range as before, modifies the value of the risk measure that reaches the value obtained in the alternative measure (see black circles in Fig. 2); i.e., in this framework model error can be completely explained as estimation error.

Furthermore, because we have in this case a complete analytical solution, we can provide one possible explanation why a numerical approach can be slow. Solving the optimisation problem (42) is equivalent to selecting the minimum of a paraboloid. A first order algorithm decreases faster in the direction of higher eigenvalues and more slowly when eigenvalues are lower. For example, Fig. 3 shows the evolution of the gradient descent numerical algorithm used in the minimisation of a quadratic function in two dimensions: the algorithm is fast in the direction with maximum variability but varies slowly in the direction of minimum variability; i.e., in the direction of the eigenvector of the matrix $\Sigma$ corresponding to the minimum eigenvalue.

In our case, for every $\eta$ in the interval of interest, we have to solve an optimisation problem. Each optimisation has two main features, as shown in (43): i) the largest eigenvalue is almost $n$ times larger than the other ones and ii) there are $n-1$ minimum eigenvalues. Thus, the numerical algorithm becomes slow in the direction of minimum variability and it could stop before it reaches the correct optimum, in particular if $n$ is very large. This can be one reason why an analytical solution can be useful.

Finally, as a second numerical example we consider a non-symmetric case. The parameters are the same ones of previous numerical example, but with mean $\mu_{i}=0.1 \cdot\left(1+x_{i}\right)$, 


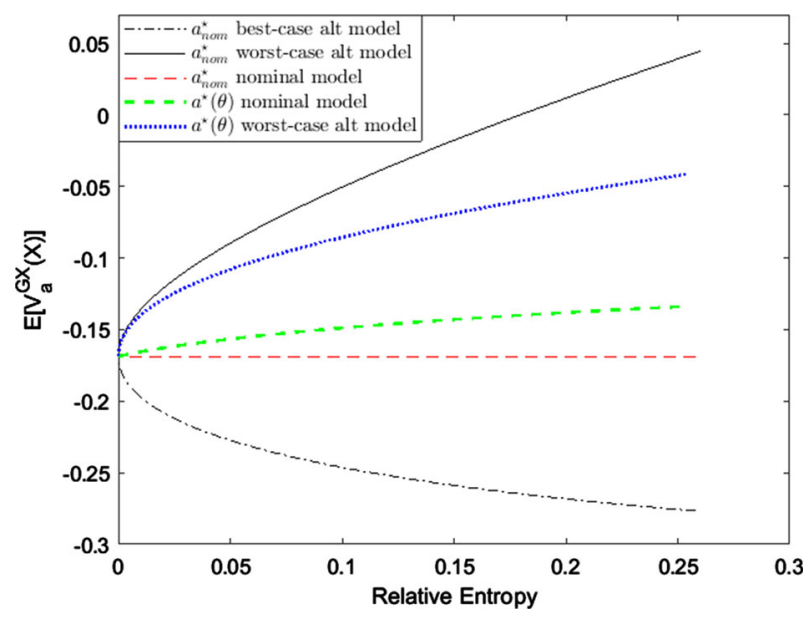

Fig. 4 Value of the risk measure versus relative entropy in a non-symmetric case. We consider $\mu_{i}=0.1 \cdot(1+$ $\left.x_{i}\right), i=1, \ldots, 10$, with $x_{i}$ drawn from a standard normal distribution, while the other parameters are the same of Fig. 2. As in Fig. 2, we have considered the relative entropy $\eta \in[0,0.25]$. The dashed red line shows the value of the risk measure in the nominal model for the optimal nominal portfolio $a_{\text {nom }}^{\star}(8)$; the black line is the value of the risk measure in the alternative model in the worst-case approach (continuous line) and in the best-case approach (dotted-dashed line) for the optimal nominal portfolio $a_{n o m}^{\star}$; the dashed green line shows the value of the risk measure in the nominal model for the robust portfolio $a^{\star}\left(\theta^{\star}\right)(38)$; the dotted blue line shows the value of the risk measure in the alternative model for the robust portfolio. (Color figure online)

$i=1, \ldots, 10$, with $x_{i}$ drawn from a standard normal random distribution. The frontier obtained is shown in Fig. 4. In this case the robust portfolio, i.e. the optimal portfolio in the worst-case alternative model $a^{\star}\left(\theta^{\star}\right)$ (38) where $\theta^{\star}$ is obtained via Proposition 11, is different from the optimal portfolio in the nominal model $a_{\text {nom }}^{\star}$, as we have proven in Proposition 13. The result obtained in Fig. 4 looks similar to the one in [5, Figure 1, p. 37] and the two plots differ just for the values of the risk measure on the vertical axis, that depend on the chosen values for $\mu$.

In Fig. 4 we can observe the consequences of selecting the robust portfolio. On the one hand, the value of the risk measure in the nominal model is larger for the robust portfolio (dashed green line) w.r.t. the one for the optimal nominal portfolio (dashed red line), where the latter clearly does not depend on the relative entropy; on the other hand, the value of the risk measure in the alternative worst-case model (with variance $\tilde{\Sigma}$ in (13)) is significantly lower for the robust portfolio (dotted blue line) w.r.t. the one valued for the optimal nominal portfolio (continuous black line).

\section{Conclusions}

We have studied the effect of model risk on the optimal portfolio in the mean-variance selection problem. Model risk is measured via the worst-case approach, taking the relative entropy as measure of the divergence between the nominal and the alternative model; in particular, we have considered all alternative models within a KL-ball $P_{\eta}$ of radius $\eta$. When asset returns are modeled with a multivariate normal, this problem has been numerically solved for small $P_{\eta}$ by Glasserman and Xu [5] in the mean-variance case with an additional constraint on the mean, chosen equal to the one in the nominal model. 
In this paper, we have analytically solved the optimal portfolio selection problem in the alternative model in a generic mean-variance framework for a generic KL-ball $P_{\eta}$. We have proven that the optimal portfolio in the worst-case approach is unique and given by Eq. (28), where the optimal $\theta^{\star}$ is the unique positive solution of the equation $R\left(\theta^{\star}\right)=\eta$ with $R(\theta)$ given in (31).

We have also solved the special case considered in Glasserman and $\mathrm{Xu}$ [5], in which they impose the alternative mean constrained (cf. solution (38) with entropy (40)).

Finally, we have analyzed in detail the situations when model risk and estimation risk coincide and we have shown two numerical examples. In particular, one of these examples considers exactly the same illustrative example of [5, Figure 1, p. 37], without been able to reproduce their numerical solutions. This fact shows the relevance of the provided analytical solution in the worst-case approach for model risk because in some cases solving the nested optimizations (cf. problem (4)) can be a challenging operational research problem from a numerical point of view.

\section{Shorthands}

$\begin{array}{ll}\text { cf. } & \text { Compare; from Latin: confer } \\ \text { e.g. } & \text { For example; from Latin: exempli gratia } \\ \text { i.e. } & \text { That is; from Latin: id est } \\ \text { KL-ball } & \text { Ball of m where the Kullback-Leibler entropy is lower than } \eta \\ \text { p.d.f. } & \text { Probability density function } \\ \text { r.v. } & \text { Random variable } \\ \text { s.t. } & \text { Such that } \\ \text { w.r.t. } & \text { With respect to }\end{array}$

Acknowledgements The authors thank all participants to the seminar at the European Investment Bank (EIB) and conference participants to the 19th Quantitative Finance Workshop at ETH Zurich and to the SIAM Conference on Financial Mathematics and Engineering 2019 at University of Toronto. We are grateful in particular to Michele Azzone, Giuseppe Bonavolontá, Mohamed Boukerroui, Szabolcs Gaal, Maurizio Grasselli, Juraj Hlinicky, Aykut Ozsoy, Oleg Reichmann, Sergio Scandizzo, Claudio Tebaldi, Pierre Tychon for useful comments. The authors acknowledge EIB financial support under the EIB Institute Knowledge Programme. The findings, interpretations and conclusions presented in this document are entirely those of the authors and should not be attributed in any manner to the EIB. Any errors remain those of the authors.

Funding Open access funding provided by Politecnico di Milano within the CRUI-CARE Agreement.

Open Access This article is licensed under a Creative Commons Attribution 4.0 International License, which permits use, sharing, adaptation, distribution and reproduction in any medium or format, as long as you give appropriate credit to the original author(s) and the source, provide a link to the Creative Commons licence, and indicate if changes were made. The images or other third party material in this article are included in the article's Creative Commons licence, unless indicated otherwise in a credit line to the material. If material is not included in the article's Creative Commons licence and your intended use is not permitted by statutory regulation or exceeds the permitted use, you will need to obtain permission directly from the copyright holder. To view a copy of this licence, visit http://creativecommons.org/licenses/by/4.0/.

\section{Appendix A}

In this appendix we prove Lemmas 1 and 7. We also state and prove a technical Lemma, which is useful to compute the eigenvalues of the nominal variance-covariance matrix $\Sigma$ in the fully symmetric case. 
Proof of Lemma 1 For any value of $\mathbf{a}$ and $\theta$ s.t. $\exists$ a solution $m_{\theta, \mathbf{a}}^{\star}(\mathbf{X})(10)$, we get

$$
R(\theta, \mathbf{a})=\mathbb{E}\left[m_{\theta, \mathbf{a}}^{\star}(\mathbf{X}) \ln m_{\theta, \mathbf{a}}^{\star}(\mathbf{X})\right]=\theta \mathbb{E}\left[m_{\theta, \mathbf{a}}^{\star}(\mathbf{X}) V_{\mathbf{a}}(\mathbf{X})\right]-\ln \mathbb{E}\left[\exp \left(\theta V_{\mathbf{a}}(\mathbf{X})\right)\right] .
$$

We now study the behaviour of this function. First, we notice that the function is continuous $\forall \theta$ s.t. $m_{\theta, \mathbf{a}}^{\star}(\mathbf{X})$ is well-defined. Then, to evaluate the slope of the relative entropy, we compute the first derivative. Supposing to choose $m_{\theta, \mathbf{a}}(\mathbf{X})$ within a class of sufficiently regular functions, we can exchange the derivative with the expected value (see e.g. [15, Th. 4, p. 429]). We get

$$
\begin{aligned}
\frac{\partial R(\theta, \mathbf{a})}{\partial \theta} & =\mathbb{E}\left[m_{\theta, \mathbf{a}}^{\star}(\mathbf{X}) V_{\mathbf{a}}(\mathbf{X})\right]+\theta \frac{\partial}{\partial \theta} \mathbb{E}\left[m_{\theta, \mathbf{a}}^{\star}(\mathbf{X}) V_{\mathbf{a}}(\mathbf{X})\right]-\frac{1}{\mathbb{E}\left[\exp \left(\theta V_{\mathbf{a}}(\mathbf{X})\right)\right]} \frac{\partial}{\partial \theta} \mathbb{E}\left[\exp \left(\theta V_{\mathbf{a}}(\mathbf{X})\right)\right]= \\
& =\theta\left(\mathbb{E}\left[m_{\theta, \mathbf{a}}^{\star}(\mathbf{X}) V_{\mathbf{a}}^{2}(\mathbf{X})\right]-\mathbb{E}\left[m_{\theta, \mathbf{a}}^{\star}(\mathbf{X}) V_{\mathbf{a}}(\mathbf{X})\right]^{2}\right)= \\
& =\theta \operatorname{Var}_{\tilde{f}}\left(V_{\mathbf{a}}(\mathbf{X})\right),
\end{aligned}
$$

where $\operatorname{Var}_{\tilde{f}}\left(V_{\mathbf{a}}(\mathbf{X})\right)$ is the variance of $V_{\mathbf{a}}(\mathbf{X})$ in the alternative measure.

Being the variance non-negative, the sign of the derivative, i.e. the slope of the relative entropy function, depends only on the sign of $\theta$ and the relative entropy is a monotone increasing function for positive values of $\theta$, and a monotone decreasing function for negative $\theta$.

Proof of Lemma 7 The optimization problem (33) can be computed solving a Lagrangian optimization w.r.t. the Lagrangian parameter $\lambda \in \mathbb{R}^{n}$

$$
\text { (33) } \Leftrightarrow \inf _{\mathbf{a} \in \mathcal{A}} \sup _{m \in P_{\eta}}\left\{\mathbb{E}\left[m(\mathbf{X}) V_{\mathbf{a}}(\mathbf{X}]+\lambda^{T}(\mathbb{E}[m(\mathbf{X}) \mathbf{X}]-\boldsymbol{\mu})\right\}=\inf _{\mathbf{a} \in \mathcal{A}} \sup _{m \in P_{\eta}} \mathbb{E}\left[m(\mathbf{X}) V_{\mathbf{a}}^{\lambda}(\mathbf{X})\right]\right.
$$

with

$$
V_{\mathbf{a}}^{\lambda}(\mathbf{X}):=V_{\mathbf{a}}(\mathbf{X})+\lambda^{T}(\mathbf{X}-\boldsymbol{\mu}) .
$$

As mentioned in the introduction (cf. Eq. (4)), the problem is equivalent to

$$
\inf _{\mathbf{a}} \inf _{\theta>0} \sup _{m} \mathcal{L}^{\lambda}(\theta, \mathbf{a} ; m(\mathbf{X})) \text {. }
$$

For any given $(\theta, \mathbf{a}) \in \mathcal{D}, \theta>0$, with $\mathcal{D}$ defined in (15), we get the equation for the constraint imposing the stationarity w.r.t. $\lambda$

$$
\mathbb{E}[m(\mathbf{X})(\mathbf{X}-\boldsymbol{\mu})]=\mathbf{0},
$$

where $\mathbf{0}$ is the null vector in $\mathbb{R}^{n}$, and the optimal $m_{\theta, \mathbf{a}}^{\lambda}(\mathbf{X})$ (cf. (10)) is

$$
m_{\theta, \mathbf{a}}^{\lambda}(\mathbf{X})=\frac{\exp \left(\theta V_{\mathbf{a}}^{\lambda}(\mathbf{X})\right)}{\mathbb{E}\left[\exp \left(\theta V_{\mathbf{a}}^{\lambda}(\mathbf{X})\right)\right]} .
$$

Equation (45) on the optimal $m_{\theta, \mathbf{a}}^{\lambda}(\mathbf{X})$ becomes

$$
\begin{aligned}
\mathbf{0} & =\frac{\mathbb{E}\left[\exp \left(\theta V_{\mathbf{a}}^{\lambda}(\mathbf{X})\right)(\mathbf{X}-\boldsymbol{\mu})\right]}{\mathbb{E}\left[\exp \left(\theta V_{\mathbf{a}}^{\lambda}(\mathbf{X})\right)\right]} \\
& =\frac{\int d \tilde{\mathbf{X}} \exp \left(-\frac{1}{2} \tilde{\mathbf{X}}^{T} \tilde{\Sigma}^{-1} \tilde{\mathbf{X}}+\theta(\boldsymbol{\lambda}-\mathbf{a})^{T} \tilde{\mathbf{X}}\right) \tilde{\mathbf{X}}}{\int d \tilde{\mathbf{X}} \exp \left(-\frac{1}{2} \tilde{\mathbf{X}}^{T} \tilde{\Sigma}^{-1} \tilde{\mathbf{X}}+\theta(\boldsymbol{\lambda}-\mathbf{a})^{T} \tilde{\mathbf{X}}\right)} \\
& =\theta \tilde{\Sigma}(\boldsymbol{\lambda}-\mathbf{a})
\end{aligned}
$$


where in the last equality $\tilde{\mathbf{X}}:=\mathbf{X}-\boldsymbol{\mu}$. The above equation implies that $\boldsymbol{\lambda}=\mathbf{a}$, because $\tilde{\Sigma}$ is invertible and $\theta>0$. For this value of $\lambda$, the quantity $V_{\mathbf{a}}^{\lambda}(\mathbf{X})$ is equal to measure of risk $V_{\mathbf{a}}^{G X}(\mathbf{X})$, proving the lemma.

Lemma 14 Given a matrix $K \in \mathbb{R}^{n \times n}$ with two values, $c$ on the diagonal and $d$ extradiagonal, i.e. of the form

$$
K=\left[\begin{array}{cccc}
c & d & \ldots & d \\
d & c & \ldots & d \\
\vdots & \vdots & \ddots & \vdots \\
d & d & \ldots & c
\end{array}\right]
$$

$K$ has an eigenvalue $\lambda_{1}=d(n-1)+c$ with eigenvector with constant weights and the remaining $n-1$ eigenvalues $\lambda_{2}=\cdots=\lambda_{n}=c-d$.

Proof We write $K$ as

$$
K=d 11^{T}+(c-d) I .
$$

We simply have to find eigenvalues of $\mathbf{1 1}{ }^{T}$ because the eigenvalues of $K$ are the sum of eigenvalues of $d \mathbf{1 1}^{T}$ and $(c-d) I$. The matrix $11^{T}$ has one eigenvalue equal to $n$ corresponding to the eigenvector $1 / n$. The remaining $n-1$ eigenvalues are all 0 with eigenvectors equal to any basis of the kernel (due to the rank-nullity theorem). We then get the eigenvalues and the eigenvectors of $K$.

\section{References}

1. Boyd, S., Vandenberghe, L.: Convex optimization. Cambridge University Press (2004)

2. Calafiore, G.C., 2007. Ambiguous risk measures and optimal robust portfolios, SIAM Journal on Optimization, 18 (3), 853-877

3. Gantmacher, F.R., Kreı̆n, M.G.: Oszillationsmatrizen, oszillationskerne und kleine schwingungen mechanischer systeme, vol. 5. Akademie-Verlag (1960)

4. Gilboa, I. and Schmeidler, D., 1989. Maxmin expected utility with non-unique prior, Journal of Mathematical Economics, 18 (2), 141-153

5. Glasserman, P. and Xu, X., 2014. Robust risk measurement and model risk, Quantitative Finance, 14 (1), 29-58

6. Hansen, L.P., Sargent, T.J.: Robustness. Princeton University Press (2008)

7. Harville D.A. (1997) Matrix Algebra from a Statistician's Perspective, vol. 1. Springer, Berlin.

8. Kerkhof, J., Melenberg, B., and Schumacher, H., 2010. Model risk and capital reserves, Journal of Banking \& Finance, 34 (1), 267-279

9. Kullback, S. and Leibler, R.A., 1951. On information and sufficiency, The annals of mathematical statistics, 22 (1), 79-86

10. Lam, H., 2016. Robust sensitivity analysis for stochastic systems, Mathematics of Operations Research, $41(4), 1248-1275$

11. Li, D. and Ng, W.L., 2000. Optimal dynamic portfolio selection: Multiperiod mean-variance formulation, Mathematical Finance, 10 (3), 387-406

12. Markowitz, H., 1952. Portfolio selection, The Journal of Finance, 7 (1), 77-91

13. Merton, R.C., 1972. An analytic derivation of the efficient portfolio frontier, Journal of Financial and Quantitative Analysis, 7 (4), 1851-1872

14. Penev, S., Shevchenko, P.V., and Wu, W., 2019. The impact of model risk on dynamic portfolio selection under multi-period mean-standard-deviation criterion, European Journal of Operational Research, 273, $772-784$

15. Protter M.H., Morrey C.B.J. (2012) Intermediate Calculus. Springer, Berlin 
16. Sherman, J. and Morrison, W.J., 1950. Adjustment of an inverse matrix corresponding to a change in one element of a given matrix, The Annals of Mathematical Statistics, 21 (1), 124-127

Publisher's Note Springer Nature remains neutral with regard to jurisdictional claims in published maps and institutional affiliations. 